\title{
Mitigating Thermal Beam Damage with Metallic Coats in Low Voltage FEG-EPMA of Geological Materials
}

\author{
Stuart Kearns ${ }^{1}$, Ben Buse ${ }^{1}$, and Jon Wade ${ }^{2}$ \\ 1. School of Earth Sciences, University of Bristol, UK \\ 2. Department of Earth Sciences, University of Oxford, UK
}

Field emission gun electronprobe microanalysis (FEG-EPMA) provides the analyst with the opportunity to characterize very small volumes of material. Using a low voltage source the irradiated volume is submicron. It is well known that some geological phases e.g. volcanic glass, carbonates, phosphates, hydrous silicates suffer beam damage under high beam current condition [e.g.1,2] and several authors suggest mechanisms either to mitigate beam damage (e.g. cryostage cooling, reduced beam currents, metallic coats etc. $[2,3,4.5]$ ) or to use the time dependent intensity to interpolate the count rate back to the original composition at zero time [5].

Under FEG-EPMA low voltage conditions, the beam power density in the irradiated volume is particularly high, compared with a 15 or $20 \mathrm{kV}$ source thus exacerbating beam damage. Here, we investigate the potential for using metallic coats (principally gold and silver) on a range of materials, glass, carbonate and phosphate to reduce various modes of beam damage (ion migration, volatilization, and sample degradation) and consider thermal models of the irradiated materials.

Figure 1 illustrates the scope of beam damage in a fluorapatite (Durango). In a carbon-coated sample, it is characterized by the formation of a physical crater, apparently depicting and therefore exploiting some crystallographic orientation. In contrast a gold coated sample shows carbon contamination buildup but no crater development so the damage is less severe. The effects of beam exposure on the X-ray emission of the two coated samples is different with both coats exhibiting fluorine ion migration at the same rate whereas only the carbon coated sample reveals a significant fall in the phosphorus signal over time. In the case of the gold coated sample, phosphorus analysis is straightforward and it is possible to use a time-dependent intensity (TDI) correction to obtain the true composition of fluorine.

Modelling the thermal system within the analytical volume through a metallic coat is complex. Results are shown in figure 2. In the case of a glass sample, as might be encountered in the analysis of a small volume melt inclusion, the cooling effect of a silver metal coat is demonstrated by low temperature increase within the sample and a broad thermal profile conducting heat through the overlying silver coat. In contrast the carbon coated sample shows a more concentrated temperature rise around the point of beam impact clearly focusing the heat to a small volume where sample damage will result; meanwhile the silver coat mitigates thermally driven beam damage. The calculated temperatures are seemingly low -apparently a few degrees which seems at odds with the observed damage. However the temperature rise is consistent with other experimental data and models e.g. [6,7] and geological materials with few exceptions have low thermal conductivities so we might expect a strong time dependency with the continual bombardment of beam electrons to the same volume - glasses for example can undergo an incubation period where beam damage is negligible until the sample heats up - possibly tens of seconds. 
Although metallic coats may be deployed to successfully mitigate beam damage, other parameters such as charge implantation at depth driving ion migration (as in the case of fluorine in apatite) and the effects the absorption of emitting X-rays need to be carefully considered.

\section{References}

[1] Reed S.J.B., Electron Microprobe Analysis and Scanning Electron Microscopy in Geology, (Cambridge Univeristy Press, Cambridge) (2005)

[2] Morgan G.B.,VI, and London, D. Am. Mineral. 81 (1996) p1176-1185

[3] Jercinovic and Williams Am. Mineral 90, (2005) p526-546

[4] Smith M.P. J. Sed Petrol. 56 (1986) p560-561

[5] Neilsen C. and Sigurdsson H., Am. Mineral. 66 (1981) p547-552

[6] Hulinsky, V et al, Microchimica Acta supp. (1996) p325-332

[7] Castaing R., (1951) Ph D. Thesis, University of Paris

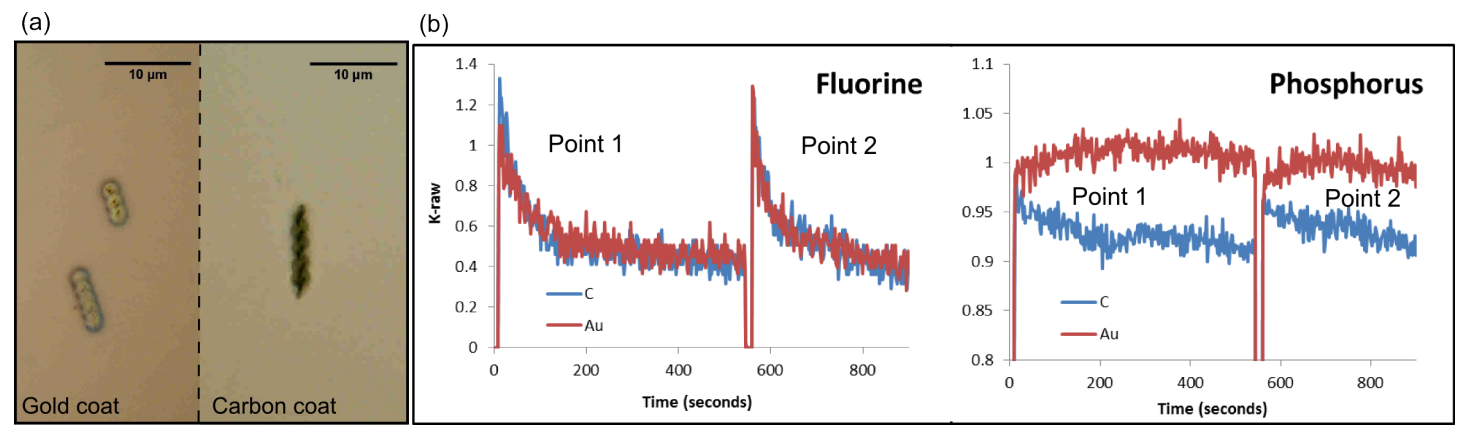

Fig 1 - a) Optical light micrographs of gold and carbon coated samples. b) TDI plots for fluorine and phosphorus in Durango Apatite contrasting the effect of different coats on the X-ray intensity.
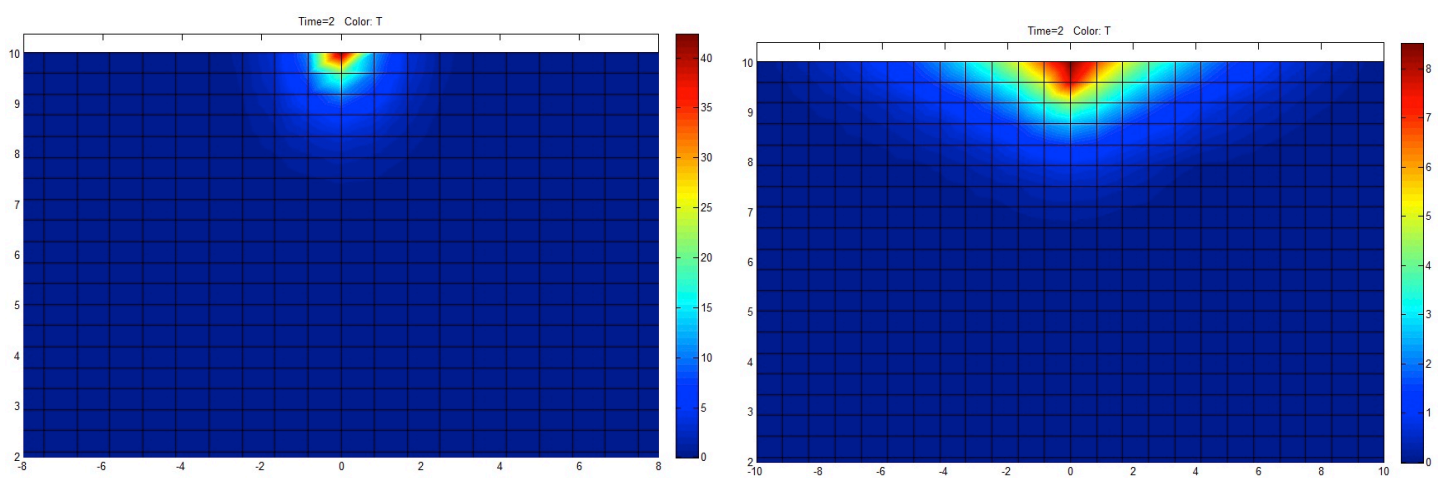

Figure 2 - Thermal models (temperature) for $2 \mathrm{~s}$ beam exposure at $5 \mathrm{kV}, 10 \mathrm{nA}$ in a volcanic glass. a) with a $20 \mathrm{~nm}$ carbon coat, b) $20 \mathrm{~nm}$ silver coat. Note the silver coat is much more efficient at cooling the sample such that the maximum temperature rise is only one fifth of that for a carbon coated sample. 